\title{
The Elevator Fragment Technique of Ilizarov in the Tibial Bone Nonunion with Bone Loss
}

\author{
Hamza Benameur*, Marwane Abouchane, Mohamed Fahsi, Mostafa Fadili, \\ Mohamed Nechad \\ Department of Orthopedic Surgery, Ibn Rochd Hospital, Casablanca, Morocco \\ Email: "Hamza.benameur@gmail.com, abouchane123@gmail.com,fahsiabdo@gmail.com, \\ M.Fadilis@hotmail.com, M.nechad@hotmail.com
}

Received 15 October 2015; accepted 4 November 2015; published 10 November 2015

Copyright (C) 2015 by authors and OALib.

This work is licensed under the Creative Commons Attribution International License (CC BY). http://creativecommons.org/licenses/by/4.0/

\section{(c) (i) Open Access}

\begin{abstract}
The management of nonunions with significant loss of bone remains a very difficult challenge for orthopedic surgeons. Several techniques have been developed, for some with difficult realization, and without convincing result for others. Our study involved two patients between 2012 and 2015, whom developed a tibial bone nonunion with significant loss of bone and having benefited from the elevator fragment technique using the Ilizarov external fixator. The evolution was good with filling of the bone loss and consolidation of the proximal osteotomy and the tibial nonunion with normal-oriented tibia. The filling of big post-traumatic bone losses by bone segmental transport, initially proposed by Ilizarov, finds its place in the reconstruction of the members, especially the tibia. This method is superior to other reconstruction techniques, in preserving the bone structure and equalizing its length. This technique has been adapted to be better tolerated by patients and more accessible to surgical teams, specifically the optimization of unilateral fixators for bone transport.
\end{abstract}

\section{Keywords}

Nonunion, Bone Loss, Elevator, Fragment, Ilizarov, Tibia

Subject Areas: Orthopedics

\section{Introduction}

Diaphyseal nonunion with significant bone loss is one of the most feared complications by orthopedic surgeons. This loss of bone substances depleting the grafted bone mass and thereby making them difficult to filling, may consider amputation.

${ }^{*}$ Corresponding author.

How to cite this paper: Benameur, H., Abouchane, M., Fahsi, M., Fadili, M. and Nechad, M. (2015) The Elevator Fragment Technique of Ilizarov in the Tibial Bone Nonunion with Bone Loss. Open Access Library Journal, 2: e2063.

http://dx.doi.org/10.4236/oalib.1102063 
The external Ilizarov fixator was invented in 1951 in Siberia, by the orthopedic surgeon Gavriil Abramovich Ilizarov, who was inspired by a shaft bow harness on a horse carriage. The concept of Ilizarov [1] was indicated firstly for length inequality (35\%), followed by the angular corrections (15\%) then by septic nonunions (15\%) [2]. In addition to its contribution in the treatment of pseudarthrosis requiring the compression to allow consolidation, the Ilizarov method also permits filling nonunion with bone loss by the elevator technique.

Our study involved two patients with tibial pseudarthrosis with significant loss of bone, which have benefited from the elevator technique using the Ilizarov external fixator between 2012 and 2015 with 1 year of decline. The purpose is to detail the technique, present our results and discuss them in the light of literature.

\section{Clinical Case}

1) Patient 1:

A 24 years old male, victim of a road accident 3 years ago (motorcyclist hit by a car), causing an open fracture of the distal leg type 2 according to Cauchoix and Duparc classification, on its antero internal face. This case was basically treated by the Hoffman external fixation. The evolution was marked by the appearance of an early sepsis requiring several sequestrectomy and long-term antibiotic therapy. The evolution after eight months left as a legacy a septic nonunion of the distal leg with bone loss of about 3 centimeters and a precarious state of the skin with scarred fistulae (Figure 1 and Figure 2).

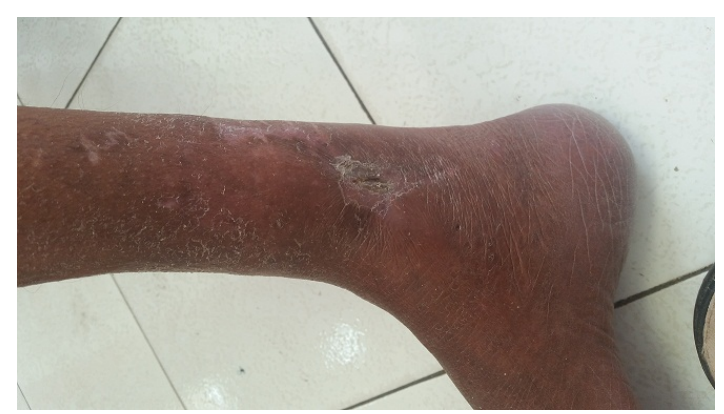

Figure 1. Patient 1 before treatment with Ilizarov technique.

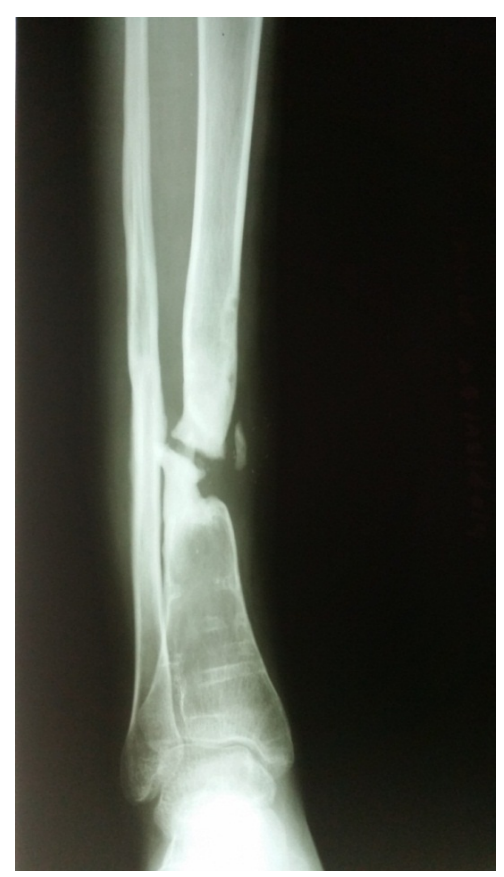

Figure 2. Radiography of the patient 1 before treatment with ilizarov technique. 
2) Patient 2:

A 28 years old male, victim of a road accident 3 years ago (motorcyclist collided with another motorcycle), causing an open fracture of the distal leg type 3 of Cauchoix and Duparc classification, at its anterolateral face. This case was initially treated by the Hoffman external fixation, associated with a cover flap. The evolution was marked by the appearance of an early sepsis requiring several sequestrectomy and long-term antibiotic therapy. The evolution after nine months left as a legacy a septic nonunion of the distal leg with bone loss of about 4 centimeters and a precarious state skin with scarred fistulae (Figure 3 and Figure 4).

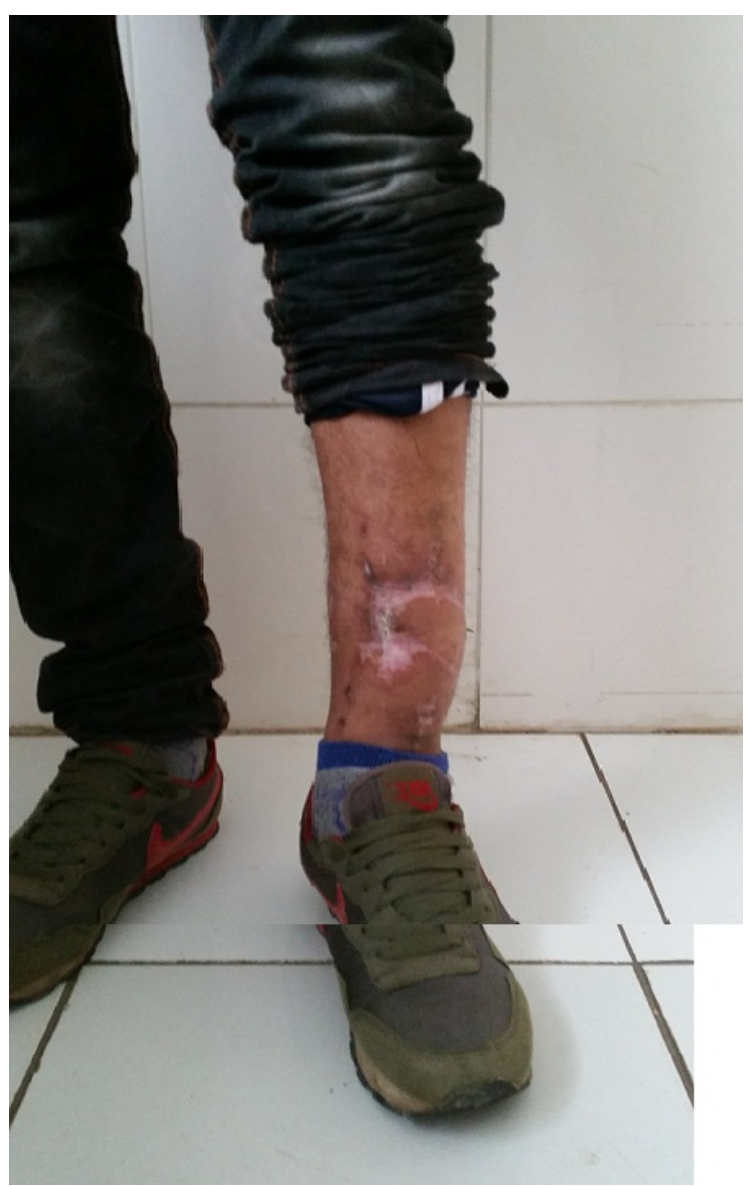

Figure 3. Patient 2 before treatment with Ilizarov technique.

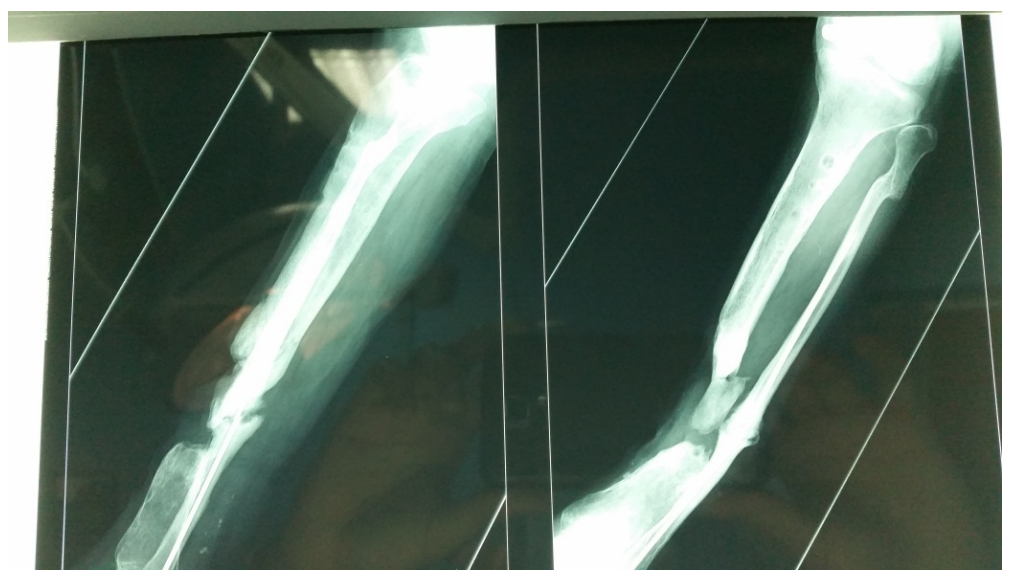

Figure 4. Radiography of the patient 2 before treatment with Ilizarov technique. 
The 2 patients benefited of the elevator fragment technique invented by Ilizarov, about two months after removal of the external fixator ( 1 year after the initial trauma).

Our technique is based on the establishment of a 5 rings external Ilizarov fixator, mounted on 3 bars, with 2 K-wires 20/10 by ring, 2 rings at the proximal epiphyseal area, upstream of the metaphyseal subperiosteal osteotomy, one ring at the cylindrical elevator fragment and two rings at distal epiphyseal zone, downstream of the nonunion and combined with an osteotomy of the fibula. The K-wires do not pass through the fibula (Figure 5).

The intermediate ring was lowered by $1 \mathrm{~mm}$ per day (one turn per day) during 40 days for the first patient (Figure 6) and 30 for the second patient (Figure 7).

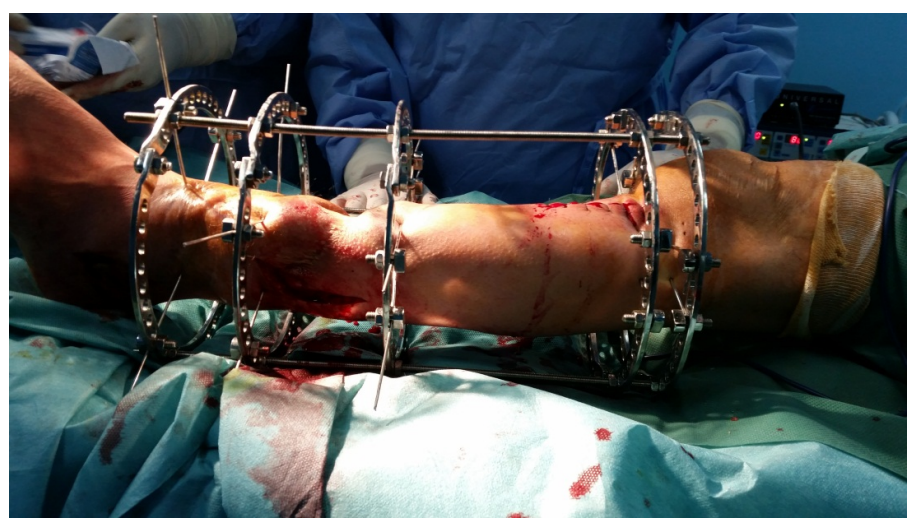

Figure 5. The elevator fragment technique of Ilizarov.

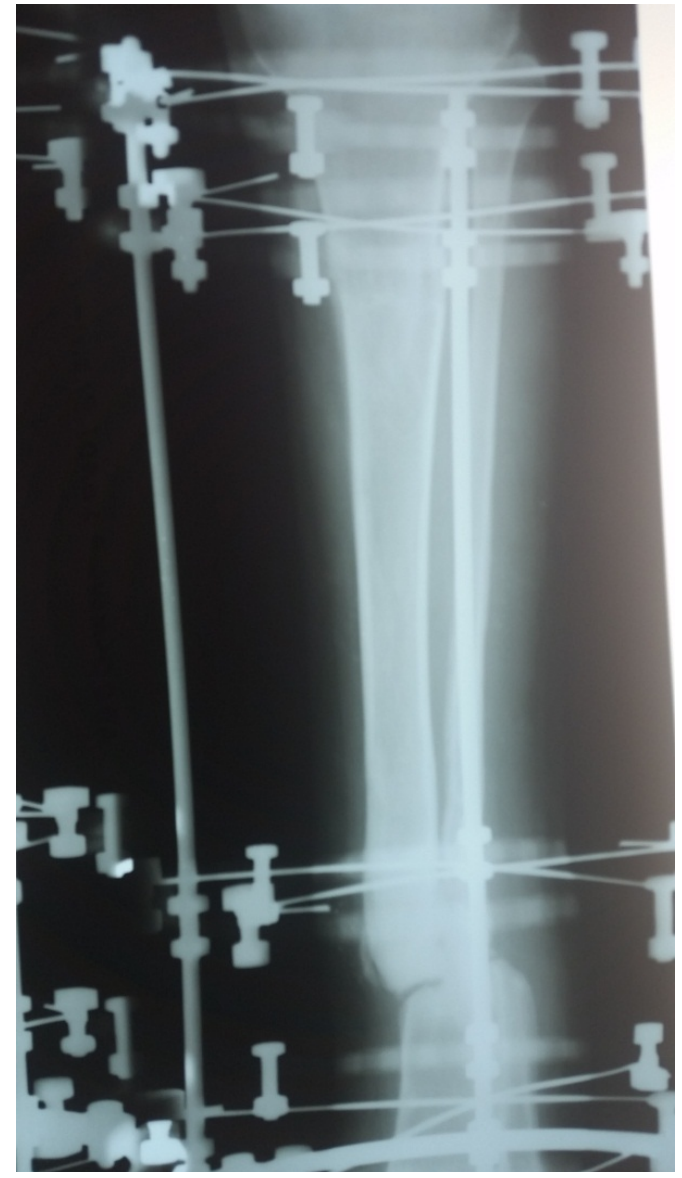

Figure 6. Patient 1 after 20 days of Ilizarov technique. 


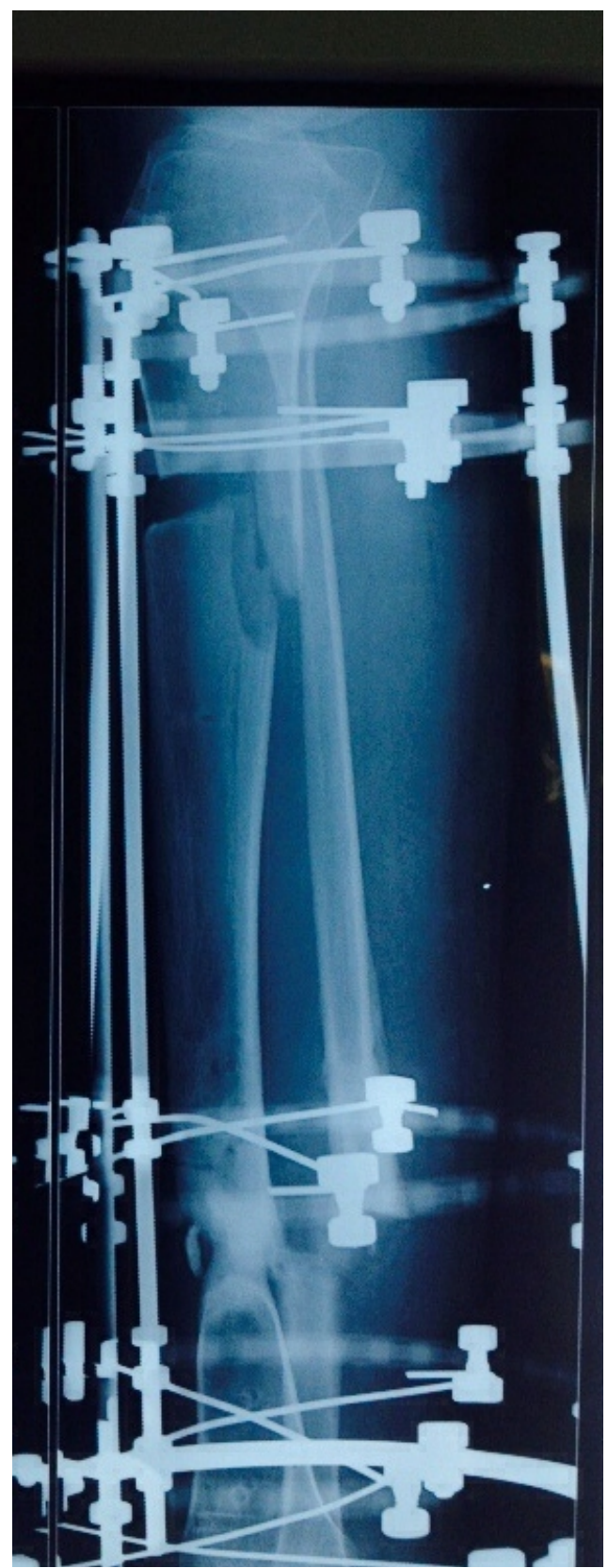

Figure 7. Patient 2 after 30 days of Ilizarov technique.

Weight bearing was immediately indicated after installation of the external Ilizarov fixator.

\section{Results}

After about 1 year of decline, the evolution was good with filling of the bone defect and consolidation of the proximal osteotomy and the nonunion bone (Figure 8 and Figure 9).

The external fixator was removed after a 7 months average (Figure 10 and Figure 11).

No shortening in 2 patients.

\section{Discussion}

The technique of the elevator is based on four principles [3], the circular external fixation, the original endosteal 


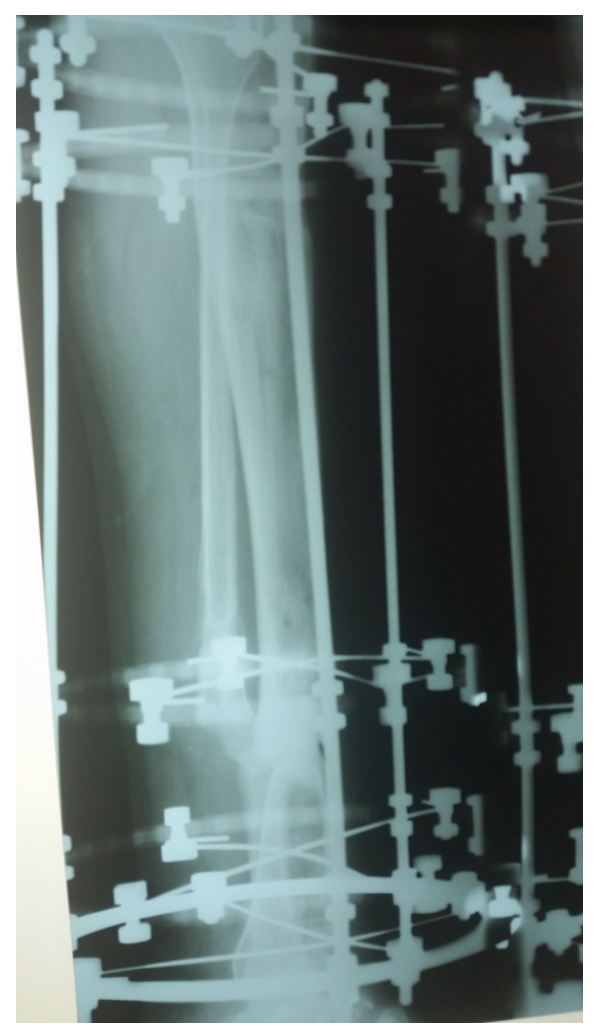

Figure 8. Patient 1 after 40 days of Ilizarov technique.

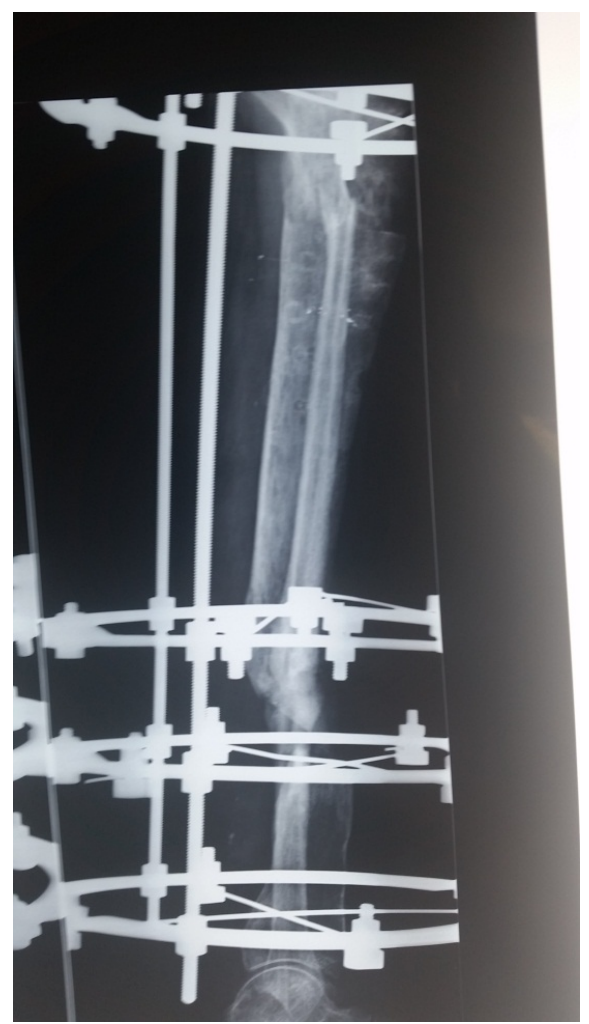

Figure 9. Patient 2 after 50 days of Iliazrov technique showing the radiological consolidation. 


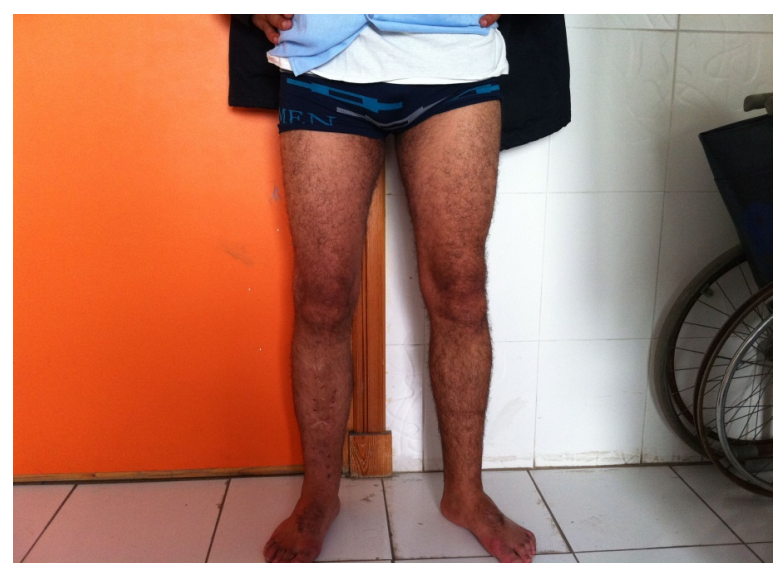

Figure 10. Patient 1 after the release of the ilizarov fixator showing the clinical consolidation.

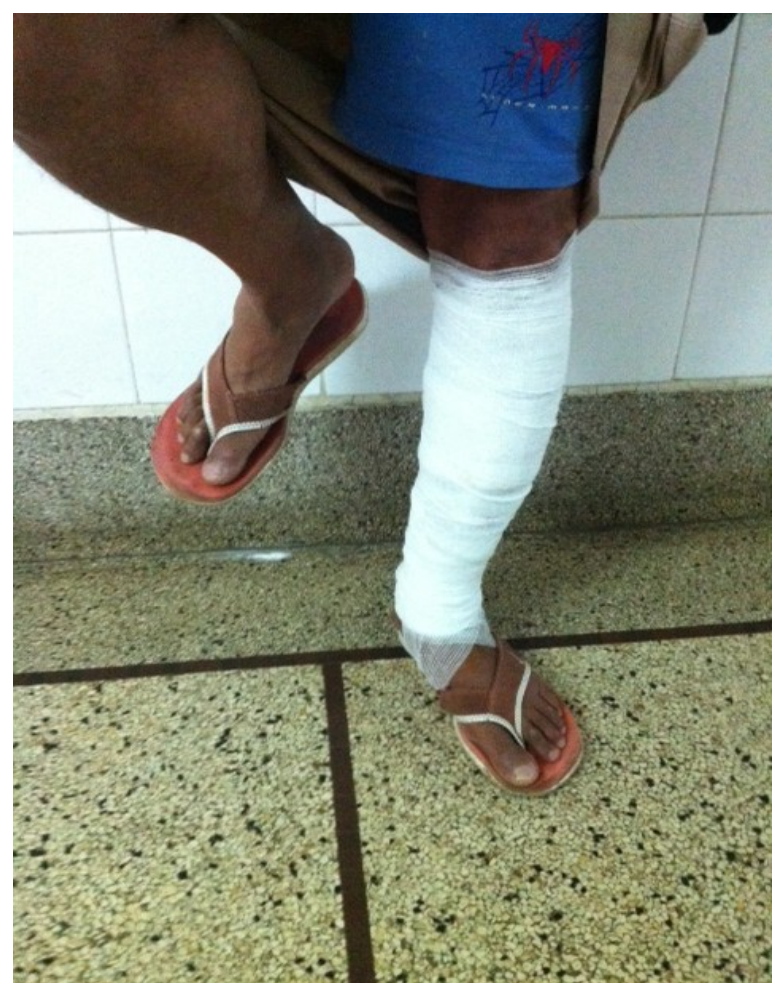

Figure 11. Patient 2 after the release of the Ilizarov fixator showing the clinical consolidation.

bone regeneration, the distraction osteogenesis, the concept of Ilizarov itself.

The principle of technique [4] is to release a third cylindrical fragment by a transverse metaphyseal osteotomy. This osteotomy, called corticotomy, respects the periosteal and endosteal vascular contributions. Using an external fixator, the third fragment is transported within the segment with its tibialis muscle-periosteum environment and neurovascular toward the opposite end of the defect. Upstream of the released fragment, under the effect of distraction, the regenerated bone fills the defect. Downstream, the compression set of the released fragment in contact with the pseudoarthrosis may allow consolidation.

Whether for a bone resection, a septic nonunion fracture or a Cauchoix-Duparc type 3 open fractures, the bone transport technique is designed for bone loss greater than 4 centimeters, with impossibility of making a vascularized bone graft, associated with the need for early weight bearing [5]. 
The literature search showed equivalence of results between the different techniques (bone graft in situ, vascularized fibula, bone segmental transport) in terms of duration of treatment, duration of consolidation and duration of wearing an external fixator, with almost identical functional results [6].

In other studies, up to 14 centimeters of bone at a single corticotomy could be regenerated, whatever the size of the fragment [4]. The use of bone graft in situ was able to appear as the failure of a conservative technique of bone. This is often imposed or carried out in principle.

As with all surgical techniques, the elevator technique has specific limits.

Complications related to regenerate bony site is dominated by plastic deformation of the regenerate causing malalignment and fractures.

Complications related to the compression site are most common and most difficult to treat. They are marked by the risk of malalignment of the two ends.

To overcome this complication, the original technique advised to set up an intramedullary K-wire guide. Other teams use an intramedullary nail locked at both ends wherein the bone segment will slide.

These complications require frequent readjustments of the orientation of the ring supporting the mobilized fragment.

Instabilities and stiffness of the joints related to movement of the insertion of the muscles and tendons and to the increase stroke of tendons remains significant. Near the ankle, an immobilization of the forefoot is frequently associated to prevent equine. In our study, early weight bearing of patients with total plantar support has helped to prevent stiffness and equinus.

Transportation can also pose specific problems as a hypertrophic bulge in the opposite site of compression zone, scar tissue interposition between fragments may stops consolidation. The use of a spacer fragment which permits a progressive ablation is an original solution to maintaining a space at the filling of a large defect.

All coverage problems are indeed not solved by this technique and flaps are often required before, during and after the transfer.

The circular fixators are rarely compatible with the realization of a flap, unilateral fixators find here great interest.

The advantages of the elevator technique are the relative compliance of bone grafted, the superiority in terms of recovery of limb length, the risk of infection decreases by less frequent iterative interventions and limited approaches, the resumption of an early weight bearing promoting consolidating and patient autonomy.

Bone regeneration allows, firstly, a larger bone resection interventions and therefore more effective in septic lesions. On the other hand, gains of fabric cover during impaction of the nonunion.

The main disadvantages of the technique remains its painfulness [7]: physical and mental pain, intolerance of transfixing K-wires, hardware footprint, limiting muscle running, etc. However, the realism of conservation of the member must be assessed case by case (Table 1 ).

\section{Conclusion}

The filling of post-traumatic big bone losses by segmental bone transport, initially proposed by Ilizarov, finds its place in the reconstruction of the members, especially the tibia. This method is superior to other reconstruction techniques in the field of preservation of bone and equalization length, although the consolidation durations are comparable to other techniques, as well as the rate of failure and complications. This technique has been adapted to be better tolerated by patients and more accessible to surgical teams, including the optimization of unilateral fixators for bone transport.

\section{Table 1. Advantages and disadvantages of the elevator fragment technique.}

\begin{tabular}{ll}
\hline Advantages & \multicolumn{2}{l}{ Disadvantages } \\
\hline - Relative compliance of bone grafted & - Physical pain \\
- Recovery of limb length & - Mental pain \\
- Less frequent iterative interventions and limited approaches & - Intolerance of transfixing K-wires \\
- Early weight bearing & - Hardware footprint \\
- A larger bone resection & - Limiting muscle running \\
- Gains of fabric cover & \\
\hline
\end{tabular}




\section{Declaration of Interest}

The authors declare that there are no conflicts of interest.

\section{Contributions of Authors}

All authors have read and approved this manuscript.

\section{The Patients Approved the Case Report}

These case reports gain the consent from ethics committee.

\section{References}

[1] Ilizarov, G.A. and Lediaev, V.I. (1992) The Replacement of Long Tubular Bone Defects by Lengthening Distraction Osteotomy of One of the Fragments. Clinical Orthopaedics and Related Research, 280, 7-10. http://dx.doi.org/10.1097/00003086-199207000-00002

[2] Caton, J., Cattaneo, R., Damsin, J.P., et al. (1995) Bilan des observations de l’Asamif. Revue de Chirurgie Orthopedique, 81, 645-649.

[3] Meyrueis, J.P. and Merloz, P. (1996) Fixation externe du squelette. Cahiers d'enseignement de la SOFCOT no. 8. Expansion Scientifique Francaise, Paris.

[4] Bauer, B., et al. (2012) Réparations tissulaires à la jambe. Springer-Verlag, France, 85-94. http://dx.doi.org/10.1007/978-2-287-99066-3_9

[5] Paley, D. and Maar, D.C. (2000) Ilizarov Bone Transport Treatment for Tibial Defects. Journal of Orthopaedic Trauma, 14, 76-85. http://dx.doi.org/10.1097/00005131-200002000-00002

[6] Prokuski, L.J. and Marsh, J.L. (1994) Segmental Bone Deficiency after Acute Trauma. Orthopedic Clinics of North America, 25, 753-763.

[7] McKee, M., Yoo, D. and Schemitsch, E.H. (1998) Health Status after Ilizarov Reconstruction of Post-Traumatic Lower Limb Deformity. Journal of Bone \& Joint Surgery (British Volume), 80, 360-364. http://dx.doi.org/10.1302/0301-620X.80B2.8192 